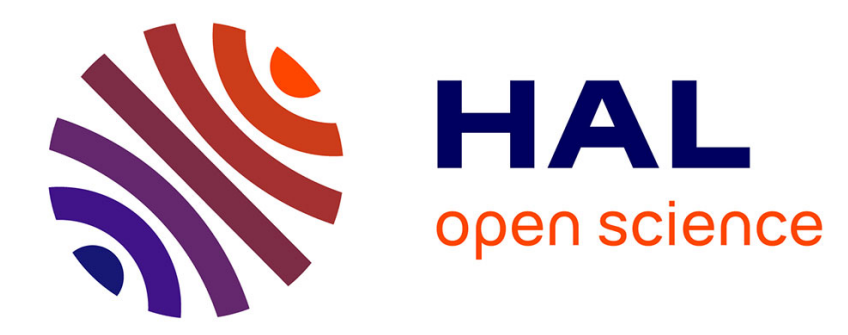

\title{
Les modèles formels et l'explication en sciences sociales
}

Nathalie Bulle

\section{To cite this version:}

Nathalie Bulle. Les modèles formels et l'explication en sciences sociales. Année Sociologique, 2005, 55 (1), pp.19-34. halshs-00137186

\section{HAL Id: halshs-00137186 \\ https://shs.hal.science/halshs-00137186}

Submitted on 17 Mar 2007

HAL is a multi-disciplinary open access archive for the deposit and dissemination of scientific research documents, whether they are published or not. The documents may come from teaching and research institutions in France or abroad, or from public or private research centers.
L'archive ouverte pluridisciplinaire HAL, est destinée au dépôt et à la diffusion de documents scientifiques de niveau recherche, publiés ou non, émanant des établissements d'enseignement et de recherche français ou étrangers, des laboratoires publics ou privés. 


\section{LES MODELES FORMELS ET L'EXPLICATION EN SCIENCES SOCIALES}

Nathalie Bulle, « Les modèles formels et l'explication en sciences sociales », L'Année sociologique, 2005, 55, n ${ }^{\circ}$, p.19-34. 


\section{LES MODELES FORMELS ET L'EXPLICATION EN SCIENCES SOCIALE}

On oppose dans cette article deux modes de connaissance du monde social, un mode descriptif attaché au réalisme des effets, et un mode explicatif attaché au réalisme des causes. Le premier s'appuie sur la modélisation de phénomènes totaux complexes, sans égard aux processus réels en jeu. Le second s'appuie sur la modélisation des processus réels, sans chercher à reproduire les phénomènes totaux observables. L'impossibilité pratique de rendre compte simultanément de manière réaliste des causes et des effets des processus sociaux réels, permet d'éclairer le problème des critères de la validité des modèles en sciences sociales.

In this article two modes of knowing the social world are opposed, a descriptive one linked to realism of effects, and an explicative one linked to realism of causes. The first is based on models of total complex phenomena, without any consideration to the real processes at play. The second is based on models of real processes, with no aim to reproduce total observed phenomena. The practical impossibility to translate realistically, both causes and effects of real social processes permits one to clarify the problem of the criteria of validity of models in social sciences.

La pensée scientifique appliquée à l'analyse empirique de la vie sociale est sujette à des illusions dont il est difficile de mesurer toute l'ampleur. Il n'est pas absurde à cet égard de rapprocher ces effets du processus idéologique à l'origine de la vision fausse que l'homme se fait naturellement de luimême selon Marx et Engels. D'après les auteurs de L'Idéologie allemande, ce dernier ne se rendrait pas compte que ses moyens de compréhension du monde, les idées, les représentations ou les concepts, sont les produits du monde réel, des rapports sociaux concrets, et non l'inverse. Mais Marx et Engels n'ont pas échappé, on le sait, aux pièges tendus à l'analyste de la vie sociale par ses propres instruments d'examen et de mesure. Ils ont substitué un causalisme structurel au causalisme idéel qu'ils dénonçaient. La dualité artificielle base-superstructure qui est au cœur de la mise au point théorique qu'ils soutiennent - la conscience de l'homme et son histoire sont déterminés par les conditions de la vie matérielle - utilise une métaphore architecturale qui mystifie aussi bien le jeu des processus sociaux «réels » en cause. Cet exemple emprunté à la pensée sociale du XIX ${ }^{\mathrm{e}}$ siècle est significatif des pièges tendus à la pensée théorique par ses propres moyens d'analyse.

La pensée formelle, en raison des exigences même de clarification auxquelles elle souscrit, définit des ensembles abstraits, des classes (genre, âge, origine sociale, appartenance religieuse, tendances politiques etc.) qui regroupent des caractères concrets hétérogènes. Dans le domaine des statistiques, 
l'analyste étudie formellement l'influence de ces ensembles abstraits, les classes définies, sur les éléments qui les composent, les individus statistiques. La réalité sociale lui apparait- la métaphore optique de Marx et Engels prend ici tout son sens - comme les reflets du monde sensible dans l'œil de l'homme : elle se présente renversée sur la tête. Les effets se donnent pour les causes.

Les propriétés observées au niveau des individus statistiques, traduites par des relations entre variables abstraites, ont une signification uniquement descriptive. Les relations diffèrent à chaque changement de niveau d'analyse parce que ces effets sont en général issus de réseaux causaux complexes. Si l'on passe directement des liens statistiques à l'interprétation sociologique, on tombe dans le piège du réalisme : on fait implicitement l'hypothèse que la relation observée au niveau des individus statistiques reflète les relations existant au niveau des individus réels.

La recherche des causes réelles des phénomènes sociaux, autrement dit l'explication sociologique, ne vise pas pour autant à transcrire la complexité de la réalité concrète. L'individu statistique de l'analyste et l'individu ideal-typique du sociologue sont tous deux des constructions abstraites. Le premier condense le réel observable. Il rend compte des effets totaux lisibles au niveau des groupes abstraits. Le second simplifie le réel. Il exprime les tendances centrales des comportements individuels étant données les structures qui organisent et contraignent les actions individuelles.

Comment passer des phénomènes totaux observés à l'explicitation de leurs causes réelles ? Dans quelle mesure ces causes exhumées se donnent pour réelles dès lors qu'on n'accède qu'à des combinaisons de leurs effets? Bref, comment l'explication sociologique est-elle possible ? Pour offrir des éléments de réponse à ces questions, deux pôles dans l'ensemble des modèles de l'action humaine sont distingués. L'un des pôles est relatif aux modèles à vocation descriptive, dont la finalité est le réalisme des effets. Le second pôle est relatif aux modèles à vocation explicative, dont la finalité est le réalisme des causes. Le problème de la différenciation pratique de ces deux pôles, de l'impossibilité de rendre compte simultanément de manière réaliste des causes et des effets des processus sociaux réels, s'ouvre sur la question de la validité des modèles en sciences sociales.

\section{La nature des modèles de l'action sociale}

Un modèle scientifique offre une représentation sélective et symbolique d'un phénomène empirique (système ou processus). La notion de modèle s'applique aussi à l'exemple type d'un corpus théorique: dans le domaine particulier de la logique mathématique, le modèle est un champ de réalisation de ce qui est exprimé par un système formel. Suivant la première acception, celle qui nous intéresse, on parle de modèle dès lors qu'une réalité que l'intelligence peut manipuler est confrontée analogiquement à une autre réalité afin d'en rendre compte de manière économique et scientifiquement exploitable.

Au sens étroit, un modèle en sciences est défini en termes mathématiques. La formalisation permet d'étudier le fonctionnement de l'ensemble réel transcrit en le soumettant à des mesures et à des tests, 
et de faire varier les hypothèses. D’une manière plus générale, mais non exclusive (il peut virtuellement être constitué d'éléments de toute nature, matérielle ou idéelle), un modèle est constitué de concepts et de relations. Plus les modèles sont formalisés, plus les concepts mis en jeu répondent à la notion de concepts scientifiques. Les concepts scientifiques se définissent par des «paramètres » qui sont détachés des objets, c'est-à-dire conçus pour eux-mêmes. Le mouvement propre à la formalisation tend en effet à procéder linéairement, allant de la définition à l'objet sans revenir en arrière (Grize 1962 :111). Ainsi, la spécificité des concepts scientifiques, par opposition aux notions communes, concepts quotidiens ou spontanés, est qu'ils sont définis par leurs relations aux autres concepts. Ils font partie intégrante d'un système de concepts où ils existent pour eux-mêmes, indépendamment des objets réels qu'ils dénotent par ailleurs. L'existence d'un tel système permet l'établissement de relations supra-empiriques entre éléments abstraits. (Vygotsky (1934) 1997 :317)

Ces relations, qui sous-tendent l'activité de la pensée formelle, rendent possible la construction de modèles à proprement parler. De la relative fermeture des concepts et relations sur lesquels il s'appuie dépend la possibilité de «manipuler » le modèle pour en déduire mécaniquement un ensemble de conséquences à propos du phénomène étudié. Formellement, le modèle est autonome par rapport au réel. Les éléments qu'il met en œuvre sont définis opérationnellement par les seules liaisons retenues, et exclusivement par elles, c'est-à-dire par l'ensemble des relations qu'ils entretiennent avec les autres éléments du modèle. Ils ne sont donc pas simplement isolés de l'ensemble des facteurs intervenant en réalité, mais ils sont littéralement reconstruits. Autrement dit, la signification des variations marquées par les facteurs du modèle ne renvoie pas à des variations réelles des objets que ces facteurs dénotent, mais aux relations avec les autres facteurs, c'est-à-dire à la théorie que le modèle exprime.

Parmi les facteurs causaux mis en jeu par le modèle, certains représentent des causes réelles. Il s'agit de facteurs explicatifs qui transcrivent, de manière symbolique et sélective, les processus sociaux en jeu. D'autres substituent leurs effets à ceux des facteurs réels. Ils ne transcrivent pas les processus véritables à l'origine des effets reproduits. Il s'agit de facteurs essentiellement descriptifs. La distinction de ces deux types de facteurs, qui font tous deux partie d'une reconstruction symbolique de la réalité, ne va pas de soi. Quand bien même les processus représentés ont pour objet de transcrire des processus réels, ces derniers sont résumés et reconstruits idéellement. Des facteurs abstraits substituent nécessairement leurs effets à ceux, complexes, des facteurs réels. Le modèle, en tant que représentation, est donc toujours essentiellement descriptif. Mais en satisfaisant des conditions méthodologiques définies, il peut être considéré comme explicatif, ou certaines de ses parties peuvent être considérées comme, explicatives.

Un phénomène simple peut être à la fois reproduit et expliqué. Les facteurs représentés, qui satisfont les conditions méthodologiques du réalisme causal, produisent alors les effets observés réellement. Le médecin qui a établi son diagnostic, compte tenu de son rôle professionnel, de ses connaissances médicales etc., prescrit une liste de médicaments au malade. Des actes sociaux simples 
sont à la fois reproduits et expliqués par la confrontation des motifs des acteurs à leur situation, laquelle met en jeu leur position sociale et leurs dispositions cognitives en particulier.

Mais les finalités descriptives et les finalités explicatives des modèles tendent à se distinguer dès lors que la complexité des phénomènes en jeu impose des simplifications substantielles. En effet, selon qu'on s'attache à décrire ou à expliquer, on met en œuvre deux types différents de réalisme. Dans la description et la prévision, le réalisme porte sur les effets, tandis que dans l'explication, il porte sur les causes. Dans le premier cas, les simplifications nécessaires portent sur les mécanismes, tandis que dans le second elles portent sur les faits et les données. En réalité, la pratique scientifique ne conduit pas à une distinction aussi nette des types de modèles réalisés. On peut considérer que l'ensemble de ceux-ci, au sein même de la pensée formelle, se situent plutôt entre deux pôles, l'un descriptif, l'autre explicatif.

\section{Les modèles descriptifs}

Du coté du pôle descriptif, les modèles visent à reproduire les données de l'observation afin d'en connaître le comportement d'ensemble d'un point de vue synchronique ou diachronique. Ils définissent par exemple un ensemble de relations entre les variables caractérisant l'ensemble réel étudié et évaluent les paramètres en jeu pour que ces relations transcrivent au mieux les données de l'observation. Les mécanismes réels restent alors des boites noires : les modèles tendent à reproduire les «entrées» et les «sorties» des ensembles réels étudiés sans en révéler les secrets de fonctionnement.

Les modèles statistiques représentent un exemple caractéristique des modèles descriptifs en sciences humaines. Ils visent à résumer des ensembles importants de données relatives aux unités statistiques en cause, ou «individus». Lorsque ces individus sont des personnes humaines, les modèles établissent des relations entre les caractères ou états associés aux individus des populations observées. L'une des techniques statistiques fondamentales, l'analyse de régression, permet d'établir des relations entre une variable «dépendante» et des variables «explicatives». Par exemple, la régression linéaire d'une variable «y $\mathrm{y}$ (à expliquer) par rapport à une variable « $\mathrm{x}$ » (explicative) conduit à définir l'équation de la droite située au plus près du nuage de points (individus i) définis par les valeurs $\left(\mathrm{x}_{\mathrm{i}}, \mathrm{y}_{\mathrm{i}}\right)$ prises par les deux variables (distances verticales) et à déterminer l'intensité de la corrélation des deux variables.

Lorsque, dans les analyses de régression, la variable dépendante n'est pas quantitative, mais qualitative (ses valeurs représentent des catégories nominales), on ne peut faire appel aux techniques de régression linéaire. Les régressions dites logistiques (terme qui fait référence à une hypothèse de distribution), qui s'y substituent, connaissent actuellement un développement immense dans la recherche empirique en sciences sociales. La tendance symptomatique d'un renversement holiste se 
traduit, dans l'utilisation de ces techniques, par une découpe de la réalité pour toucher au plus près de l'individu singulier, comme si les caractères à expliquer de ce dernier étaient bien les effets des causes multiples interagissantes qu'identifient les variables «explicatives». A propos d'une étude qu'elle a réalisée sur les performances scolaires, Michèle Tribalat (2001) rend compte de cette tentation, lorsqu'une variable "explicative" entre en interaction avec de nombreuses autres, de découper le champ de modélisation en fonction de cette variable. En développant la précision descriptive des situations individuelles, l'analyste nourrit l'espoir d'en élucider la logique sous-jacente : «Vouloir saisir l'individu dans toute sa diversité et entrer le plus finement dans la complexité des comportements individuels, dans l'espoir d'y trouver une cohérence cachée, illisible autrement, laquelle ouvrirait des perspectives infinies sur la compréhension de la société, semble être la tendance actuelle vers laquelle se dirige la recherche en sciences sociales. » (Tribalat 2001 : 9) Mais chaque découpage, qui permet de conduire l'analyse sur des groupes distincts au sein de la population étudiée, au lieu de simplifier les relations qu'entretiennent les variables entre elles, s'ouvre sur de nouveaux schémas d'interactions et cela, indéfiniment. Le découpage du champ découvre des complexités toujours nouvelles. L'inverse ne serait vrai que si les variables statistiques «explicatives» représentaient des causes réelles auquel cas la simplification du champ devrait tendre à en révéler les effets : «Tout bien considéré, il faut un certain aveuglement pour espérer expliquer une réalité complexe à partir de faits dont on ne voit pas, a priori, quel sens leur donner. » On étudie classiquement, remarque Tribalat, l'influence du genre, de l'origine ethnique et de la taille de la famille, sur la réussite au baccalauréat. La mise en évidence de telles influences renvoie souvent implicitement à l'idée que ces conditions constituent en elles-mêmes un avantage ou un handicap. On tend à «essentialiser» ces caractères, à considérer de manière « réaliste» l'effet des facteurs statistiques. Or, ces avantages ou handicaps «nets » ne sont que des tendances qui marquent le solde d'effets multiples si bien que les effets des variables explicatives retenues s'annuleraient si on égalisait les conditions.

Les méthodes d'analyse causale, développées par la recherche sociologique notamment entre les années 1960 et les années 1980, et qui sont apparentées aux analyses de régression, offrent un autre exemple de la tendance au renversement holiste de l'interprétation statistique. Pour étudier les données empiriques dont il dispose (données d'enquête par exemple), l'observateur élabore des modèles théoriques (définis par des équations «structurales $»^{1}$ ) qui spécifient les liens de causalité postulés c'est-à-dire d'influence - entre les variables retenues. De tels modèles permettent d'estimer la force des relations causales définies. Mais les forces relatives des relations causales, que mesurent les

\footnotetext{
${ }^{1}$ Le qualificatif de «structural» signifie ici que les paramètres et équations utilisées n'ont pas, quant aux liaisons établies entre les variables, un sens seulement descriptif, mais reflètent des relations causales invariantes. Quand les variables sont utilisées sous forme standardisée, on fait appel à des techniques de «path analysis». Dans ce cas les «paramètres structuraux» se réfèrent à des «path coefficients » et sont directement interprétables à partir des coefficients de corrélation entre les variables, le modèle structural se réfère alors à un «path model».
} 
«paramètres structuraux », ne représentent que la partie visible de l'iceberg social en cause. Elles sont évaluées à partir du jeu des covariations des variables qui caractérisent les individus statistiques ${ }^{2}$.

L'explication, au sens statistique, n'est autre que l'expression d'une dépendance fonctionnelle évaluée comme plus ou moins représentative de la réalité sur un plan essentiellement descriptif. Les relations abstraites entre états ou caractères définis au niveau des individus, établies sur des ensembles plus ou moins nombreux d'unités, sont les effets des processus sociaux sous-jacents à ces états ou caractères. Ces processus mettent en jeu des interactions entre les individus et les structures situationnelles que les modèles statistiques ne peuvent, par construction même, appréhender ${ }^{3}$. L'individu statistique n'est qu'une résultante théorique de l'agrégation des actions des individus concrets évoluant dans de multiples structures situationnelles différentes. Du point de vue statistique ces situations ne sont pas différenciées, l'individu statistique évolue dans un vide social (Cherkaoui 2003). A travers lui les effets décrits se produisent de variables à variables pour rendre compte des phénomènes totaux observés. L'approche multiniveau (Courgeau 2004, 2005) réitère ce même type de relations à différents degrés. Les modèles statistiques évaluent les effets macrologiques observables de causes macrologiques abstraites, et non des causes micrologiques réelles.

\section{Les modèles explicatifs}

Du coté du pôle explicatif, au contraire, la modélisation des processus réels en cause traduit les actions d'individus «séparés », suivant l'expression même de Max Weber. Ces derniers ne sont pas porteurs passifs de qualités et d'attributs, mais acteurs interagissant dans des conditions situationnelles déterminées. Les modèles explicatifs visent à rendre compte des mécanismes et processus qui soustendent concrètement les phénomènes observés. Appliqués à caractériser des mécanismes ou des

\footnotetext{
${ }^{2}$ Les modèles mettant en jeu des ensembles de variables où des effets multiples sont considérés comme des fonctions de causes multiples. Chaque effet est représenté par une équation fonctionnelle, le système d'équations étant conçu de manière à ce qu'une variable représentant un effet dans une équation joue comme cause dans les équations ultérieures. Les relations fonctionnelles ne sont pas supposées traduire des implications logiques, mais plutôt des liens probabilistes (ce qui se traduit dans les équations par l'introduction de perturbations aléatoires). Un problème fondamental tient au rôle joué par la «clôture » (self-containment) du système d'équations fonctionnelles qui constitue le modèle à tester. Cette condition est satisfaite seulement si aucun facteur implicite n'affecte à la fois des variables explicatives et des variables expliquées. Dans le cas contraire, les estimations des paramètres structuraux sont biaisées. En effet, l'action d'un facteur implicite sur différentes variables explicatives et expliquées mesurées, par le jeu même des covariations à partir desquelles sont estimés les paramètres structuraux, est attribuée aux variables causales du modèle.

3 " The statistical study is concerned with the attributes of individuals, though not with attributes of particular individuals, but with attributes of which we know only that they are possessed by a certain quantitatively determined proportion of all the individuals in our «collective» or «population». (...) The collectives of statistics, on which we study the regularities produced by the "law of large numbers", are thus emphatically not wholes in the sense in which we describe social structures as wholes. This is best seen from the fact that the properties of the collectives with statistics studies must remain unaffected if from the total of elements we select at random a certain part. Far from dealing with structures of relationships, statistics deliberately and systematically disregards the relationships between the individual elements. (...) the consequence of this is that in the statistical study of social phenomena the structures with which the theoretical social sciences are concerned actually disappear.” F.A.Hayek (1952: p.107-108)
} 
processus opérant véritablement, les modèles théoriques tendent à isoler des relations causales particulières et à s'abstraire des phénomènes totaux observables. Dans une optique essentiellement explicative, un modèle ne retient que les éléments nécessaires à la représentation des relations élémentaires en cause.

Le modèle du dilemme du prisonnier de la théorie des jeux, celui du marché des vieux clous de George Akerlov (1970) ou le modèle de ségrégation spatiale de Thomas Schelling (1971) sont des exemples types de modèles purement explicatifs et, peut-on ajouter, sont considérés comme des modèles de modèles, des exemples types de modèles théoriques. Chacun d'eux montre que le phénomène social représenté par lui peut être expliqué par certains facteurs causaux essentiels.

La structure d'interaction caractérisée par le «dilemme du prisonnier » est la plus célèbre de la théorie des jeux. Elle permet d'expliquer que des stratégies qui, au niveau des groupes d'individus concernés sont sous6optimales, soient choisies rationnellement par des acteurs sociaux ne pouvant se concerter ${ }^{4}$.

Le modèle d'Akerlov est à l'origine du développement de toute une branche de l'économie, l'économie de l'information. Ce modèle explique, à partir d'une formalisation simple, pourquoi des transactions économiques sont condamnées en raison de problèmes d'asymétrie d'information. L'évaluation des véhicules par les acheteurs de véhicules d'occasion, et donc le prix qu'ils sont prêts à payer, est fonction de la qualité moyenne des voitures proposées à la vente. Comme les vendeurs ont intérêt à mettre sur le marché des biens de qualité inférieure ou égale à cette évaluation, la qualité moyenne des biens mis sur le marché diminue et, avec elle, la taille du marché. Akerlov souligne l'intérêt de l'extrapolation de son modèle à de multiples phénomènes sociaux. Très généralement le modèle explique pourquoi certaines relations sociales sont condamnées parce que les attentes, réglées sur des caractères évalués plus ou moins subjectivement au niveau d'un groupe d'acteurs sociaux, défavorisent individuellement toute une partie des membres de ce groupe. D'après l'économiste cette

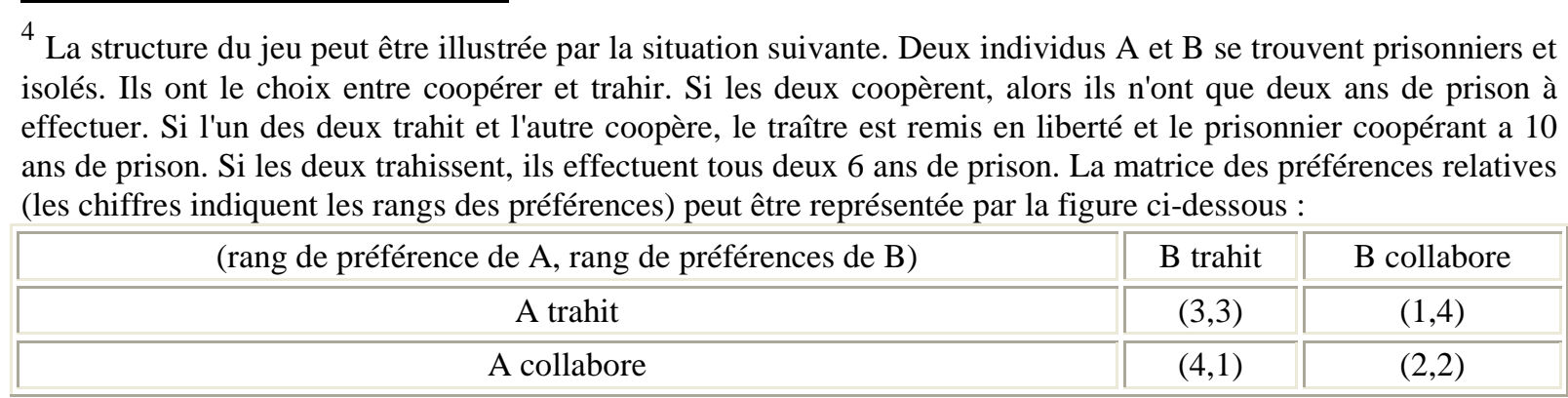

Si l'on suppose (comme on le fait en règle générale pour ces jeux simples) que $\mathrm{A}$ et $\mathrm{B}$ ne peuvent pas communiquer, leur doit se faire dans l'incertitude. Si chacun d'eux maximise son gain minimal (stratégie « maximin »), tous deux trahissent. On dit que cette stratégie est dominante parce qu'elle est choisie par les joueurs rationnels. C'est une situation d'« équilibre », c'est-à-dire une situation dans laquelle aucun joueur n'a intérêt à modifier son comportement compte tenu du comportement attendu de l'autre joueur. Or c'est une situation qui est collectivement sous optimale en regard d'un choix concerté (celui où les individus collaborent tous deux). Boudon (1979) décrit différentes applications sociologiques de ce système d'interaction. 
asymétrie de l'information sociale justifie de nombreuses institutions qui délivrent des certificats, garanties symboliques qui diminuent l'incertitude des échanges. Elle révèle en particulier l'importance de l'institution scolaire pour soutenir l'emploi des minorités : les sanctions scolaires évitent que les compétences d'un candidat ne soient évaluées subjectivement en fonction des compétences moyennes observées dans sa communauté d'appartenance.

Schelling explique, à l'aide du mouvement de pièces sur un damier, que des différenciations sociales ou spatiales fortes peuvent être engendrées par des aversions individuelles tempérées pour les situations minoritaires. Le modèle simule un phénomène d'amplification des différenciations groupales (il s'agit ici de la composition ethnique des voisinages) sur la base de l'interdépendance des décisions individuelles. Les choix de sortir ou d'entrer dans les groupes (voisinages) dépendent de leur composition (ethnique) et contribuent progressivement à modifier à la fois groupe quittés et groupes rejoints. Les différenciations groupales tendent alors au cours du processus à surpasser les motivations sous-jacentes ${ }^{5}$.

Les mondes dans lesquels agissent les acteurs d'Akerlov ou de Schelling ne sont pas des simplifications du monde réel, ce sont des mondes imaginaires d'où a disparu toute particularité qui ne servirait pas à rendre compte des relations causales en jeu. Les auteurs de ces modèles ne font d'ailleurs aucun effort pour confronter leurs résultats à la réalité. Ils ne proposent pas d'hypothèses qui puissent être testées à cet égard. Ils ne font qu'identifier de manière informelle les cas de phénomènes sociaux observables où les facteurs causaux qu'ils mettent en évidence sont susceptibles d'opérer. Leurs modèles transposent certains traits du monde réel dans un monde «possible » au sens où les causes sont réelles mais non pas les situations. Les modèles d'Akerlov et de Schelling, comme exemples de modèles explicatifs purs, mettent en lumière des «tendances » du monde réel. Une façon de comprendre la notion de tendance ici est de se représenter les phénomènes du monde réel comme les produits de l'interaction de multiples facteurs causaux. Une «tendance » peut être interprétée comme le jeu d'un petit sous-ensemble de ces facteurs ${ }^{6}$. Les phénomènes complexes observés sont alors expliqués qualitativement, de manière analogique en référence au modèle. Or plus ce dernier augmente en généralité et est susceptible de rendre compte de phénomènes différents, plus il présente une réalité épurée. Le potentiel explicatif s'oppose à cet égard au réalisme empirique.

\section{La simulation des processus sociaux réels}

Entre ces deux extrêmes, les modèles purement descriptifs et les modèles purement explicatifs, il existe tous les cas de modèles comportant des hypothèses essentiellement descriptives qui visent

\footnotetext{
${ }^{5}$ La possibilité des déplacements est matérialisée sur le damier par l'existence de cases vides. La première distribution des pièces est aléatoire. Les variables sont ici le nombre d'individus dans chaque groupe, l'intensité de la sensibilité qui est à l'origine des comportements discriminatoires, la définition des voisinages et les règles de procédure déterminant l'ordre et la destination des déplacements.

${ }^{6}$ Cf. Sugden (2002).
} 
seulement à transcrire les résultats de processus réels complexes, et des hypothèses explicatives qui visent à mettre en lumière des relations causales effectives. C'est cette association d'hypothèses descriptives et d'hypothèses explicatives qui sous-tend la simulation des processus sociaux réels. Ce que l'on entend par simulation s'apparente à la modélisation dynamique. La simulation est plus précisément appliquée à transcrire l'évolution diachronique de systèmes réels : « une simulation imite un processus par un autre processus » (Hartmann 1996). Elle est fondée sur la définition de processus théoriques qui visent à rendre compte des caractères émergents de processus sociaux complexes. Appliquée aux processus sociaux réels, la simulation peut allier au potentiel descriptif offert par l'imitation d'effets empiriquement observables, le potentiel explicatif que lui confère la mise en oeuvre de relations causales effectives.

Dans la plupart des modèles visant à rendre compte de la dynamique d'agrégation des actions individuelles, la modélisation des décisions des acteurs sociaux tend à reproduire les résultats des processus décisionnels, mais ces processus ne sont pas objets de la simulation, ils ne sont pas expliqués par le modèle. Les comportements individuels mettant en jeu des relations entre motivations et données situationnelles sont alors expliqués par l'analyste et décrits par le modèle, l'explication formelle à proprement parler portant sur la dynamique du phénomène social en cause. Le modèle proposé par Boudon (1973) dans L'inégalité des chances s'appuie sur les effets d'interaction, constatés empiriquement, qui existent entre les variables relatives à la réussite scolaire, aux milieux sociaux et aux décisions d'orientation. Le modèle permet en particulier de rendre compte de la différenciation des taux de scolarisation en fonction des origines sociales: les différences décisionnelles, en se réitérant à chaque étape des parcours individuels, ont des effets multiplicatifs, de nature exponentielle, sur les inégalités sociales.

Les relations mises en évidence par des modèles théoriques généraux font partie intégrante d'autres modèles appliqués à l'explication de phénomènes plus spécifiques. Les modèles d'Akerlov et de Schelling éclairent les processus de valorisation des sections au sein du système éducatif (Bulle 1999). La logique de l'action collective d'après Mancur Olson (1966), qui explique pourquoi les groupes latents (sans instance représentative) ayant intérêt à la production d'un bien collectif tendent à ne pas agir pour la favoriser, ou le processus d'expansion scolaire qui intervient dans le modèle de Boudon (1973), peuvent être interprétés tous deux par des structures de type dilemme du prisonnier explicitées en théorie des jeux.

\section{La validité des modèles dans les sciences sociales en général et en sociologie en particulier}

Quels critères permettent-ils d'estimer la validité des modèles, leur pertinence descriptive ou explicative ? Ce problème apparaît d'autant plus clairement que, pour rendre compte d'une réalité 
complexe, le réalisme des causes tend à s'opposer au réalisme des effets. Autrement dit, l'adéquation aux données de l'observation ne permet pas de juger la pertinence explicative des modèles, tandis que le réalisme causal des hypothèses tend à contrarier leurs potentialités descriptives.

Milton Friedman (1953), dans un essai abondamment commenté, a proposé une réponse à cette question dans le cadre de la théorie économique. Quel est le critère, demande Friedman, permettant de dire si un écart par rapport au réalisme est acceptable ou non, sachant que le réalisme total est inatteignable? La réponse de l'économiste est considérée comme hétérogène et ambiguë, au point que des lecteurs aux préférences intellectuelles différentes sont susceptibles d'y voir chacun une confirmation de leur propre point de vue (Mäki 2003). La thèse centrale de Friedman est néanmoins bien explicite. Les modèles économiques, avance-t-il, ne doivent pas être jugés sur la vraisemblance de leurs hypothèses, mais sur leur pouvoir prédictif.

Un exemple proposé par Friedman concerne la modélisation des tirs d'un maître de billard. On peut considérer que l'individu agit comme s'il connaissait les formules mathématiques complexes des coups. Cette hypothèse est irréaliste, mais elle conduit à des prévisions recevables. Selon l'économiste, on peut considérer qu'une hypothèse (i.e. une théorie) est composée de deux parties. La première partie correspond au modèle abstrait plus «simple» que le monde réel. La seconde partie spécifie la catégorie de phénomènes pour lesquels le modèle est une représentation adéquate, ainsi que la correspondance entre les variables du modèle et les phénomènes observables. Le critère de validité du modèle est, sur cette base, le test par la prévision.

Friedman s'intéresse aux qualités descriptives ou prévisionnelles (au réalisme des effets) des modèles. Mais la seconde partie de la théorie qui spécifie le champ de validité de la théorie et, plus précisément, la correspondance entre représentations formelles et circonstances concrètes, est là pour « contenir » le modèle dans ses limites représentatives et prévenir tout débordement. Cette condition place sur un même plan formel les éventuelles parties explicatives du modèle assurant le «réalisme des causes » et ses éventuelles parties descriptives assurant le « réalisme des effets », tout au moins ne les différencie-t-elle potentiellement que par leur potentiel d'extrapolation (spécifié par les conditions de correspondance).

Dans l'essai de Friedman, le test par la prévision, le réalisme des effets, n'a de valeur pour l'évaluation du modèle qu'assorti du champ d'application des hypothèses. En l'occurrence, dès lors qu'il sert les mêmes objectifs cognitifs et permet d'atteindre un même degré d'approximation de la réalité, un modèle serait préférable à un autre, non parce que ses hypothèses seraient plus réalistes au sens empirique (précision descriptive), mais parce que son champ d'application s'avèrerait plus vaste (pertinence analytique). On voit que si le réalisme causal n'a pas un rôle « direct » à jouer dans la modélisation économique, son rôle indirect, par l'intermédiaire du champ d'application des théories, est potentiellement très important. Friedman s'appuie sur l'exemple de la modélisation de la densité des feuilles sur un arbre. L'économiste propose l'hypothèse suivante. Chacune des feuilles est positionnée «comme si », étant donné la position de ses voisines, elle cherchait délibérément à 
maximiser, et cela conformément aux lois physiques permettant de choisir une position optimale, la quantité de lumière qu'elle reçoit. L'économiste remarque que le résultat obtenu par une adaptation passive aux circonstances externes (sous l'effet de l'action de la lumière solaire sur le feuillage) est en pratique le même que celui qui aurait été obtenu par une adaptation délibérée à celles-ci. Or si l'hypothèse alternative de l'adaptation passive ici est plus attirante que l'hypothèse d'une adaptation délibérée c'est, d'après Friedman, non parce que ses «postulats» sont plus réalistes, mais parce qu'elle fait partie d'une théorie plus générale s'appliquant à une plus grande variété de phénomènes

Les problèmes posés en sciences humaines visent cependant, en général, la compréhension des phénomènes. Dans cette optique, l'objet premier de la modélisation n'est pas de faire « coïncider » les modèles construits avec la réalité qui est celle des effets. Le test par la prévision ne peut assurer des qualités explicatives des modèles. La question est alors de savoir s'il existe un critère permettant d'apprécier la justesse des relations causales mises en œuvre. Cette question est d'autant plus délicate que les éléments des modèles ne sont, comme on l'a vu, jamais « réalistes » au sens propre. Les modèles sont fermés alors que les processus sociaux sont essentiellement ouverts. Le modélisateur s'expose à cet égard au risque de ne déduire de son modèle que ce qu'il y a mis. Les simplifications qu'il opère peuvent aussi produire des artefacts. Les catégories construites, la simultanéité des décisions, l'information des individus, leurs interrelations, la géométrie et la taille des populations etc., peuvent fausser les conclusions qu'il tire du modèle en terme de compréhension des phénomènes. Certaines simplifications sont néanmoins purement mathématiques. Par exemple, la modélisation des effets de l'agrégation de décisions individuelles nombreuses permet d'ignorer les influences spécifiques qui les sous-tendent, tant que ces dernières ne sont pas systématiques, pour ne retenir que la « tendance centrale » des actions.

Pour apprécier la robustesse explicative d'un modèle, il est nécessaire de pouvoir séparer méthodologiquement les hypothèses descriptives (réalisme des effets) et les hypothèses explicatives (réalisme des causes). Cette distinction est possible seulement si ces hypothèses peuvent être tenues pour relativement autonomes au regard de la réalité figurée, dans le cadre de la théorie développée. Cette condition peut être tenue comme faisant partie intégrante de la précision du champ d'application des hypothèses dans l'optique friedmanienne. Plus précisément ici, dans un but d'explication du phénomène social représenté, elle prévient le jeu de facteurs implicites sur les facteurs explicatifs du modèle. Les éléments essentiellement descriptifs doivent en effet pouvoir être développés dans la perspective du réalisme causal sans invalider le noyau explicatif du modèle. Les critères méthodologiques de l'explication, qui dépendent d'options théoriques préalables sont alors applicables aux hypothèses explicatives. Les conditions de validité empirique, adaptées en fonction des finalités descriptives du modèle, permettent enfin d'apprécier si les hypothèses avancées sont conformes aux données de l'observation.

\footnotetext{
${ }^{7}$ Si donc l'essai de Friedman a réellement encouragé à l'excès le formalisme en matière de théorie économique ce serait pour avoir été mal interprété. Cf. à ce sujet D.W. Hands (2003)
} 


\section{BIBLIOGRAPHIE}

Akerlov, G.A., 1970. The market for "lemons": quality uncertainty and the market mecanism, Quaterly Journal of Economics, n84, p.488-500.

Boudon R., 1973, L'inégalité des chances. La mobilité sociale dans les sociétés industrielles, Paris, Armand Colin.

Boudon R., 1979, La logique du social, Paris, Hachette.

Bulle N., 1999, La rationalité des décisions scolaires, Paris, PUF.

Cherkaoui M., 2003. The individual and the collective, European Review, vol.11, nº4, p.489-504.

Courgeau D., 2004, Du groupe à l'individu. Synthèse multiniveau, Editions de l'Institut National Démographique, Paris.

Courgeau D., 2005, "General introduction" in D.Courgeau (dir.), Methodology and Epistemology of Multilevel Analysis, Boston, Kluwer Academic Publishers.

Friedman M., 1953, «The Methodology of Positive Economics » in Essays in Positive Economics, Chicago, Chicago University Press, p.3-43.

J.B.Grize «Remarques sur les limitations des formalismes » in E.W.Beth, J.B.Grize \& al., Implication, formalisation, logique naturelle, Paris, PUF, 1962, p.104-127.

Hands, D.W., 2003. "Did Milton Friedman's methodology license the Formalist Revolution?” Journal of Economic Methodology, 10, 4, p.507-520.

Hartmann S. "The world as a process” in Hegselmann R., U.Mueller, K.G.Troitzsch K.G. (eds.) 1996, Modelling and simulation in the social sciences from the philosophy of science point of view, London, Kluwer Academic Publishers.

Hayek F.A., (1952), 1979, The Counter-Revolution of Science, Indianapolis, Liberty Fund Inc.

Mäki U., 2003. “'The methodology of positive economics' (1953) does not give us the methodology of positive economics" Journal of Economic Methodology, 10,4, p.495-505.

Olson M. (1966), 1978, Logique de l'action collective, Paris, PUF.

Schelling, T.C., 1971. Dynamic models of segregation, Journal of Mathematical Sociology, Vol.1, p.143-186. 
Sugden R., 2002, "Credible worlds: the status of theoretical models in economics", in U.Mäki (ed.), Fact and Fiction in Economics: Models, Realism, and Social Construction, Cambridge, Cambridge University Press, p.107-136.

Tribalat M., 2001, « Modéliser pour quoi faire? »INED, fascicule n 97.

Vygotski L. (1934), 1997, Pensée et langage, Paris, SNEDIT. 